\section{Lazer sedentário e consumo de alimentos entre adolescentes brasileiros: Pesquisa Nacional de Saúde do Escolar (PeNSE), 2009}

\author{
Sedentary leisure time and food consumption \\ among Brazilian adolescents: the Brazilian \\ National School-Based Adolescent Health \\ Survey (PeNSE), 2009
}

Lidyane do Valle Camelo 1

Jôsi Fernandes de Castro Rodrigues 1

Luana Giatti 1

Sandhi Maria Barreto 1
${ }^{1}$ Faculdade de Medicina, Universidade Federal de Minas Gerais, Belo Horizonte, Brasil.

Correspondência L. V. Camelo

Programa de Pós-graduação em Saúde Pública, Faculdade de Medicina, Universidade Federal de Minas Gerais. Av. Professor Alfredo Balena 190, Belo Horizonte, $M G$ 30130-100, Brasil. lidyanecamelo@gmail.com

\section{Abstract}

The objective of this paper was to investigate whether sedentary leisure time was associated with increased regular consumption of unhealthy foods, independently of socio-demographic indicators and family context. The analysis included 59, 809 students from the Brazilian National School-Based Adolescent Health Survey (PeNSE) in 2009. The response variable was sedentary leisure time, defined as watching more than two hours of TV daily. The target explanatory variables were regular consumption of soft drinks, sweets, cookies, and processed meat. Odds ratios (OR) and 95\% confidence limits (95\%CI) were obtained by multiple logistic regression. Prevalence of sedentary leisure time was 65\%. Regular consumption of unhealthy foods was statistically higher among students reporting sedentary leisure time, before and after adjusting for sex, age, skin color, school administration (public versus private), household assets index, and household composition. The results indicate the need for integrated interventions to promote healthy leisure-time activities and healthy eating habits among young people.

Sedentary Lifestyle; Food Consumption; School Health; Adolescent

\section{Introdução}

As mudanças biológicas, sociais e de comportamento fazem parte da adolescência e afetam de forma significativa os hábitos alimentares dessa parcela populacional. Nessa fase os adolescentes se tornam mais independentes; comportamentos alimentares adquiridos nessa época da vida tendem a influenciar a dieta na vida adulta 1 .

Hábitos alimentares poucos saudáveis estão associados a inúmeros agravos à saúde, entre eles, a obesidade, cuja prevalência triplicou entre os meninos e mais do que dobrou entre as meninas com idade entre 10 e 19 anos nas duas últimas décadas 2 .

A permanência por longos períodos diários em frente à televisão (TV) tem sido associada à obesidade e ao sobrepeso em crianças e adolescentes em estudos transversais $3,4,5,6,7,8$ e longitudinais 9,10 em diversos países. Acredita-se que maior tempo em frente à TV contribua para o ganho de peso por dois mecanismos: menor gasto energético $11,12,13$ e aumento do consumo de alimentos de alto teor calórico 11,14,15,16,17,18,19. Tais alimentos são excessivamente veiculados pelos comerciais, que também podem induzir à concepção equivocada a respeito do seu valor nutricional. Além de consumirem mais alimentos calóricos, as crianças de famílias que assistiam à TV durante duas ou mais horas parecem consumir menos frutas e hortaliças 16. Esses são alguns dos motivos que sustentam a recomendação da 
Organização Mundial da Saúde (OMS) 20 de reduzir o tempo diário em frente à TV a menos de duas horas.

O ambiente familiar é importante para o controle do lazer sedentário 21 . Longos períodos em frente à TV têm sido associados à baixa escolaridade materna 22 , viver em lar monoparental 23 e em ambiente onde não há restrição ao uso de mídias 24.

O objetivo do presente estudo foi investigar se tempo diário em frente à TV superior a duas horas está associado ao consumo regular de refrigerantes, guloseimas, biscoitos doces e embutidos independentemente de características sociodemográficas e de composição familiar em escolares brasileiros.

\section{Metodologia}

\section{Tipo de estudo e população}

Trata-se de um estudo transversal que utilizou dados da primeira Pesquisa Nacional de Saúde do Escolar (PeNSE), um inquérito realizado com escolares da $9^{a}$ série das redes pública e privada de ensino do país, nos meses de maio a junho de 2009, nas 26 capitais estaduais e no Distrito Federal 25.

A amostra probabilística estratificada foi obtida por meio de seleção em dois estágios. Os estratos amostrais corresponderam às capitais das Unidades da Federação e ao Distrito Federal, totalizando 27 estratos. Em cada um desses estratos, as escolas foram agrupadas segundo sua dependência administrativa (pública ou privada) e o tamanho da amostra foi alocado proporcionalmente em cada um desses grupos 25 .

A coleta de dados foi realizada nas salas de aula de forma anônima e individual, por meio de questionário estruturado, autoaplicável, utilizando-se um computador de mão, o Personal Digital Assistant (PDA). O questionário foi organizado em módulos temáticos que incluíam características sociodemográficas e do contexto familiar, e também a exposição a diversos fatores de risco e proteção para a saúde. A participação do aluno foi voluntária e o escolar podia se abster de responder a qualquer pergunta. A PeNSE foi aprovada pelo Comitê Nacional de Ética em Pesquisa do Ministério da Saúde sob o parecer n ${ }^{\circ}$. 11.537. Maiores detalhes sobre a metodologia do inquérito foram descritos por Malta et al. 26.

\section{Variáveis do estudo}

A variável resposta no presente trabalho foi o lazer sedentário definido como o tempo diário em frente à TV superior a duas horas (sim, não). Essa informação foi obtida pela pergunta: "Num dia de semana comum, quantas horas por dia você assiste à TV?", que tinha as seguintes opções de respostas: "não assisto à TV"; "menos de 1 hora por dia"; "cerca de 1 hora por dia"; "cerca de 2 horas por dia”; "cerca de 3 horas por dia”; "cerca de 4 horas por dia"; "cerca de 5 horas por dia"; "cerca de 6 horas por dia" e; "cerca de 7 ou mais horas por dia”.

As variáveis explicativas de maior interesse neste estudo foram o consumo regular de alimentos não saudáveis: refrigerantes, guloseimas (balas, bombons, chicletes, doces, chocolates ou pirulitos), biscoitos doces e embutidos (hambúrguer, salsicha, mortadela, salame, presunto, $n u$ ggets ou linguiça). Consumo regular foi definido como o consumo em pelo menos cinco dos sete dias que antecederam a pesquisa.

As variáveis sociodemográficas consideradas para ajuste foram: sexo (feminino e masculino); idade ( $\leq 13,14,15 \mathrm{e} \geq 16$ anos); cor da pele autorreferida (branca, preta, parda e outras); dependência administrativa da escola (pública ou privada); e índice de bens no domicílio, que variou de 0 a 1 e foi agrupado em tercis. Para o cálculo desse índice, foram considerados apenas os bens com prevalência menor que $70 \%$ (telefone fixo, máquina de lavar roupas, computador com Internet, carro e moto). Foram atribuídos pesos à presença de cada um desses bens que corresponderam ao complemento da frequência relativa na amostra estudada. Assim, os itens que foram menos frequentes receberam um peso maior 27.

A variável composição familiar (mora com ambos os pais, mora com somente um dos pais, não mora com nenhum dos pais) foi utilizada para ajustar as análises pelo contexto familiar.

\section{$\underline{\text { Análise }}$}

Inicialmente, foi feita a descrição do tempo diário em frente à TV de acordo com as variáveis sociodemográficas, composição familiar e consumo regular de guloseimas, refrigerantes, biscoitos doces e embutidos.

A associação entre a variável resposta e as variáveis explicativas foi mensurada por meio do teste do quiquadrado de Pearson, com nível de significância de 5\%. Posteriormente, procedeuse à análise univariada para a obtenção de odds ratio (OR) e seu intervalo de $95 \%$ de confiança (IC95\%), por meio de regressão logística. As análises brutas e ajustadas foram conduzidas separadamente para cada um dos alimentos não saudáveis enumerados anteriormente. As variáveis de ajuste foram os indicadores sociodemográficos e a composição familiar. 
A análise foi feita no software Stata, versão 10.0 (Stata Corp., College Station, Estados Unidos) utilizando o comando svy (com fatores de ponderação) apropriado para a análise dos dados obtidos pelo desenho amostral complexo. Esse procedimento permite corrigir as diferentes probabilidades de seleção de escolares em cada estrato e o efeito do delinemento da amostra.

\section{Resultados}

Entre os 60.973 escolares que participaram da PeNSE, 1.164 foram excluídos do presente estudo por não terem respondido à pergunta referente ao tempo gasto em frente à TV. Dos 59.809 adolescentes estudados, $52,7 \%$ eram do sexo feminino; $24,5 \%$ tinham 13 anos ou menos; $47,1 \%, 14$ anos; $18,2 \%, 15$ anos; $10,1 \%, 16$ anos ou mais de idade; $40,2 \%$ relataram cor da pele branca; e $79 \%$ eram alunos de escolas públicas.

Dos escolares participantes, 35\% (IC95\%: 34,3-35,7) relataram permanecer por até duas horas em frente à TV; 15,5\% (IC95\%:15,1-16,0) por três horas; 29,7\% (IC95\%: 29,1-30,3) por quatro a seis horas; e $19,7 \%$ (IC95\%: 18,9-20,5) por sete horas ou mais.

A prevalência de lazer sedentário, tempo diário em frente à TV superior a duas horas, foi de 65\% (IC95\%: 64,3-65,7). Tal prevalência foi estatisticamente maior entre os escolares do sexo feminino, de idade inferior a 13 anos, que relataram cor da pele parda, provenientes de escola pública, e que residiam com somente um dos pais e estatisticamente menor entre os alunos que residiam em domicílios localizados no terceiro tercil de índice de bens (Tabela 1).

\section{Tabela 1}

Prevalência de tempo diário em frente à TV superior a duas horas, segundo variáveis sociodemográficas e composição familiar entre escolares das capitais dos estados brasileiros e do Distrito Federal. Pesquisa Nacional de Saúde do Escolar (PeNSE), 2009.

\begin{tabular}{|c|c|c|c|}
\hline Variáveis & $\begin{array}{l}\text { Prevalência } \\
\text { \% (IC95\%) }\end{array}$ & Valor de $p$ & OR (IC95\%) \\
\hline \multicolumn{4}{|l|}{ Sexo } \\
\hline Masculino & $63,0(62,0-64,0)$ & \multirow{2}{*}{$<0,001$} & 1,00 \\
\hline Feminino & $66,8(65,7-67,8)$ & & $1,18(1,11-1,26)$ \\
\hline \multicolumn{4}{|l|}{ Idade (anos) } \\
\hline$<13$ & $66,4(65,0-67,8)$ & \multirow{4}{*}{0,006} & 1,00 \\
\hline 14 & $64,7(63,6-65,8)$ & & $0,93(0,86-0,99)$ \\
\hline 15 & $65,6(64,1-67,0)$ & & $0,96(0,88-1,04)$ \\
\hline 16 ou mais & $62,0(59,8-64,2)$ & & $0,83(0,74-0,92)$ \\
\hline \multicolumn{4}{|l|}{ Cor da pele autorreferida } \\
\hline Branca & $62,5(61,1-63,9)$ & \multirow{4}{*}{$<0,001$} & 1,00 \\
\hline Preta & $66,6(64,8-68,4)$ & & $1,20(1,08-1,32)$ \\
\hline Parda & $67,1(66,0-68,1)$ & & $1,22(1,13-1,32)$ \\
\hline Outras & $64,9(62,5-67,3)$ & & $1,11(0,97-1,26)$ \\
\hline \multicolumn{4}{|l|}{ Índice de bens } \\
\hline 1으 tercil (menor) & $67,1(65,9-68,2)$ & \multirow{3}{*}{$<0,001$} & 1,00 \\
\hline 2oo tercil & $67,4(66,2-68,6)$ & & $1,01(0,94-1,09)$ \\
\hline 3으 tercil (maior) & $60,9(59,5-62,3)$ & & $0,76(0,71-0,83)$ \\
\hline \multicolumn{4}{|c|}{ Dependência administrativa da escola } \\
\hline Privada & $59,6(58,0-61,3)$ & \multirow{2}{*}{$<0,001$} & 1,00 \\
\hline Pública & $66,4(65,6-67,2)$ & & $1,33(1,24-1,44)$ \\
\hline \multicolumn{4}{|l|}{ Composição familiar } \\
\hline Reside com pai e mãe & $63,9(62,9-64,8)$ & & 1,00 \\
\hline Reside com um dos pais & $67,4(66,2-68,5)$ & $<0,001$ & $1,16(1,09-1,25)$ \\
\hline Não reside com os pais & $61,7(58,9-64,4)$ & & $0,90(0,81-1,02)$ \\
\hline
\end{tabular}

IC95\%: intervalo de 95\% de confiança; OR: odds ratio. 
O consumo regular de guloseimas, refrigerantes, biscoitos doces e embutidos foi relatado por 50,9\% (IC95\%: 50,1-51,8), 37,2\% (IC95\%: 36,238,1), 33,5\% (IC95\%: 32,7-34,4), e 17,9\% (IC95\%: 17,3-18,5) dos adolescentes, respectivamente O consumo regular desses alimentos foi estatisticamente maior entre os escolares que relataram assistir à TV por mais de duas horas diárias (Tabela 2).

As associações entre o consumo regular de guloseimas, refrigerantes, biscoitos doces e embutidos e tempo diário de TV superior a duas horas foram estatisticamente significantes antes e após os ajustes pelas características sociodemográficas e a composição familiar (Tabela 3).

\section{Discussão}

Além da alta prevalência do hábito de assistir à TV por mais que duas horas diárias entre os escolares participantes da PeNSE, os resultados mostram que esse hábito está associado ao maior consumo regular de guloseimas, refrigerantes, biscoitos doces e embutidos independentemente de características sociodemográficas e da composição familiar. Nossos achados são preocupantes e consistentes com de outros estudos internacionais $11,14,15,16,17,18,19$.

A alta prevalência de tempo diário em frente à TV superior a duas horas também foi encontrada em vários países ocidentais, como os Estados

Tabela 2

Prevalência do consumo regular de alimentos não saudáveis ( $\geq 5$ dias/semana) segundo tempo diário em frente à TV entre escolares das capitais dos estados brasileiros e do Distrito Federal. Pesquisa Nacional de Saúde do Escolar (PeNSE), 2009.

\begin{tabular}{|c|c|c|c|}
\hline \multirow[t]{3}{*}{ Consumo regular } & \multicolumn{2}{|c|}{ Tempo diário em frente à TV superior a duas horas } & \multirow[t]{3}{*}{ Valor de $p$} \\
\hline & Não & Sim & \\
\hline & $\%$ (IC95\%) & $\%$ (IC95\%) & \\
\hline Guloseimas & $44,0(42,5-45,4)$ & $54,7(53,7-55,7)$ & $<0,0001$ \\
\hline Refrigerantes & $33,2(31,8-34,5)$ & $39,3(38,2-40,4)$ & $<0,0001$ \\
\hline Biscoitos doces & $28,0(26,8-29,3)$ & $36,5(35,5-37,5)$ & $<0,0001$ \\
\hline Embutidos & $14,7(13,7-15,7)$ & $19,6(18,8-20,4)$ & $<0,0001$ \\
\hline
\end{tabular}

IC95\%: intervalo de 95\% de confiança.

\begin{tabular}{|c|c|c|}
\hline Consumo regular de alimentos não saudáveis & OR bruto (IC95\%) & OR ajustado * (IC95\%) \\
\hline \multicolumn{3}{|l|}{ Guloseimas } \\
\hline Não & 1,00 & 1,00 \\
\hline Sim & $1,54(1,44-1,64)$ & $1,50(1,40-1,61)$ \\
\hline \multicolumn{3}{|l|}{ Refrigerantes } \\
\hline Não & 1,00 & 1,00 \\
\hline $\operatorname{Sim}$ & $1,30(1,23-1,39)$ & $1,34(1,26-1,43)$ \\
\hline \multicolumn{3}{|l|}{ Biscoitos doces } \\
\hline Não & 1,00 & 1,00 \\
\hline Sim & $1,47(1,28-1,56)$ & $1,46(1,35-1,57)$ \\
\hline \multicolumn{3}{|l|}{ Embutidos } \\
\hline Não & 1,00 & 1,00 \\
\hline Sim & $1,41(1,28-1,56)$ & $1,42(1,29-1,56)$ \\
\hline
\end{tabular}

IC95\%: intervalo de 95\% de confiança; OR: odds ratio.

* OR ajustado por sexo, idade, cor da pele autorreferida, índice de bens no domicílio, dependência administrativa da escola e composição familiar. 
Unidos, Reino Unido, Escócia, Holanda e Noruega 11. Dados da Health Behavior School-aged Children 2001/2002 (HBSC 2001/2002), pesquisa sobre comportamentos em saúde de escolares de 35 países mostram altas prevalências desse comportamento, embora com grande variação: de $40 \%$ entre os escolares da Suíça até mais de $80 \%$ entre os ucranianos 11 .

No Brasil, assim como no Canadá, Israel e Ucrânia, essa prevalência diminuiu com a idade 11. Diferentemente dos resultados do HBSC 2001/2002 11 e do Youth Risk Behavioral Survey (YRBS) 6, nos Estados Unidos, encontramos uma prevalência mais elevada de tempo em frente da TV entre escolares do sexo feminino. A associação inversa com as condições socioeconômicas é coerente com outros estudos internacionais 11,14,15,28 e pode ser resultado do menor custo da TV frente às demais opções diárias de lazer e esporte 19. A maior frequência desse hábito entre escolares que autorreferiram cor da pele como preta e parda possivelmente está correlacionado às piores condições socioeconômicas desses escolares. Nossos achados também coincidem com os relatados por Lindquist et al. 23 no que tange à composição familiar: adolescentes que vivem apenas com um dos pais apresentam maior frequência de lazer sedentário e isso pode ser em razão da menor condição de monitoramento parental adequado.

A altíssima prevalência de tempo elevado em frente à TV preocupa, em particular, pelo papel obesogênico da TV. Estudos sobre o tema mostram que esse efeito pode ser mais atribuído ao aumento da ingestão de alimentos de alta densidade energética, enquanto se assiste à TV, do que decorrente da diminuição dos níveis de atividade física 29,30 . No presente estudo, a associação entre tempo diário em frente à TV e consumo de alimentos não saudáveis não foi explicada por diferenciais de renda, conforme sugere o fato de famílias com menos recursos consumirem mais alimentos de alta densidade calórica por causa de seu custo reduzido ${ }^{31}$. Nossos resultados também evidenciam que essa associação praticamente não foi modificada após ajustes pela composição familiar.

Uma das explicações para o maior consumo de alimentos não saudáveis entre os jovens que assistem à TV por mais tempo é exposição constante dos mesmos a propagandas televisivas desses alimentos. Revisões sistemáticas de estudos sobre o tema indicam a existência de associações positivas fortes e consistentes entre exposição a propagandas de alimentos e a aquisição, o consumo e as preferências de crianças e adolescentes por determinados alimentos 32,33 . Um estudo americano mostrou que apenas trinta segundos de exposição são suficientes para influenciar as escolhas alimentares das crianças ${ }^{13}$. As propagandas de produtos alimentícios são as mais frequentes na TV aberta brasileira e os alimentos predominantemente divulgados são aqueles com altos teores de gordura e/ou açúcar e sal 34 . Monteiro et al. 35 argumentam que o marketing agressivo dos alimentos ultraprocessados, como refrigerantes, biscoitos, doces, embutidos e comida pronta, promove o consumo excessivo de calorias por reforçar a hiperpalatabilidade desses alimentos, aumentar progressivamente as normas sociais relativas ao tamanho de porções e o consumo excessivo de calorias.

A PeNSE constitui-se na maior pesquisa entre escolares já realizada no país. Como a pesquisa foi realizada apenas nas capitais e no Distrito Federal, as características dos escolares, o perfil de exposição à TV e a prevalência de consumo de alimentos não saudáveis aqui descritas podem não corresponder àquela encontrada em outras cidades do país, como as de pequeno porte por exemplo. Os adolescentes que não frequentam ou que estavam fora da escola no dia da pesquisa também foram excluídos e podem representar um grupo diferente, com piores comportamentos relacionados à saúde, conforme sugerem estudos em outros países 36,37,38,39. Também vale ressaltar que as análises realizadas no presente estudo basearam-se em informações obtidas por questionário autoaplicado e podem conter erros de aferição decorrentes de sub-relato ou por dificuldade de compreensão das perguntas pelos participantes da pesquisa. Sabemos que estar fora da escola assim como o menor grau de aptidão escolar é mais frequente entre escolares que provêm de famílias com piores níveis de renda e escolaridade. Muito embora a participação na PeNSE tenha sido elevada e a frequência à escola tenha aumentado expressivamente no país nas últimas décadas 40 , não podemos descartar que a prevalência estimada neste estudo seja um pouco subestimada, dado que há uma associação inversa entre situação socioeconômica e tempo em frente à TV.

Nos últimos anos, as análises com dados de inquéritos populacionais conduzidas no Brasil têm privilegiado a regressão de Poisson, cujo resultado é expresso em razão de prevalência. Isso se justifica, em especial, quando há uma frequência elevada da variável resposta, pois a OR superestima a razão de prevalência. No presente estudo, optou-se por utilizar a regressão logística, já que o nosso objetivo era apenas identificar associações estatísticas. Essa mesma análise utilizando a regressão de Poisson, não apresentou diferença na significância estatística das associações encontradas. Fato que corrobora 
a adequação do uso OR para atender a esse tipo de objetivo.

\section{Considerações finais}

Nossos achados sugerem que medidas públicas de promoção da saúde devem ser tomadas a fim de alterar o padrão de exposição à TV em crianças e adolescentes. Destaca-se que a escola é um am- biente propício para esse tipo de estratégia, onde atividades extraclasse poderiam ser desenvolvidas por programas escolares, de forma a que as crianças se envolvam em atividades que possam minimizar a exposição ao lazer sedentário, como o hábito de assistir à TV. Políticas públicas focadas na prática de alimentação saudável e na atividade física regular são fundamentais para reduzir e prevenir o sobrepeso e a obesidade não apenas na juventude, mas também na vida adulta.

\section{Resumo}

O objetivo deste trabalho foi investigar se o lazer sedentário está associado a um maior consumo regular de alimentos não saudáveis independentemente de indicadores sociodemográficos e de contexto familiar. A análise envolveu 59.809 escolares da Pesquisa Nacional de Saúde do Escolar (PeNSE) conduzida em 2009 A variável resposta foi o lazer sedentário, definido como o tempo diário em frente à TV superior a duas horas/dia. As variáveis explicativas de interesse foram o consumo regular de refrigerantes, guloseimas, biscoitos doces e embutidos. Odds ratios (OR) e intervalos de 95\% de confiança (IC95\%) foram obtidos por regressão logística múltipla. A prevalência de lazer sedentário foi de 65\%. O consumo regular de alimentos não saudáveis foi estatisticamente maior entre os que relataram lazer sedentário, antes e após ajuste por sexo, idade, cor da pele autorreferida, dependência administrativa da escola, índice de bens no domicílio e composição familiar. Esses resultados apontam a necessidade de intervenções que promovam, de forma integrada, lazer e dieta saudável entre os jovens.

Estilo de Vida Sedentário; Consumo de Alimentos; Saúde Escolar; Adolescente

\section{Colaboradores}

L. V. Camelo participou da idealização do artigo, trabalhou na análise e na interpretação dos dados, redigiu a primeira versão do artigo e trabalhou na sua revisão crítica. J. F. C. Rodrigues auxiliou na revisão de literatura, na redação do artigo e na sua revisão crítica. L. Giatti contribuiu na elaboração da versão final e aprovou a versão a ser publicada. S. M. Barreto participou da idealização do artigo, contribuiu para a sua versão final e aprovou a versão a ser publicada.

\section{Agradecimentos}

Ao Ministério da Saúde pelo financiamento, à Fundação de Amparo à Pesquisa do Estado de Minas Gerais (FAPEMIG) pelas bolsas de pós-graduação, à L. V. Camelo e J. F. C. Rodrigues, à Coordenação de Aperfeiçoamento de Pessoal de Nível Superior pela bolsa concedida à L. Giatti pelo Programa de Apoio a Projetos Institucionais com a Participação de Recém-Doutores (PRODOC-CAPES) e ao Conselho Nacional de Desenvolvimento Científico e Tecnológico (CNPq; processo $\mathrm{n}^{\circ}$. 300159/99-4) pela bolsa de produtividade em pesquisa de S. M. Barreto. 


\section{Referências}

1. Story M, Neumark-Sztainer D, French SA. Individual and environmental influences on adolescent eating behaviors. J Am Diet Assoc 2002; 102(3 Suppl):S40-51.

2. Veiga GV, Cunha AS, Sichieri R. Trends in overweight among adolescents living in the poorest and richest regions of Brazil. Am J Public Health 2004; 94:1544-8

3. Crespo C, Smit E, Troiano R, Bartlett S, Macera C, Andersen R. Television watching, energy intake, and obesity in US children results from the third National Health and Nutrition Examination Survey, 1988-1994. Arch Pediatr Adolesc Med 2001; 155:360-5.

4. Lowry R, Wechsler H, Galuska DA, Fulton JE, Kann L. Television viewing and its associations with overweight, sedentary lifestyle, and insufficient consumption of fruits and vegetables among US high school students: differences by race, ethnicity, and gender. J Sch Health 2002; 72:413-21.

5. Tremblay M, Willms J. Is the Canadian childhood obesity epidemic related to physical activity? Int J Obes Relat Metab Disord 2003; 27:1100-5.

6. Fleming-Moran M, Thiagarajah K. Behavioral interventions and the role of television in the growing epidemic of adolescent obesity: data from the 2001 Youth Risk Behavioral Survey. Methods Inf Med 2005; 44:303-9.

7. Fonseca VM, Sichieri R, Veiga GV. Fatores associados à obesidade em adolescentes. Rev Saúde Pública 1998; 32:541-9.

8. Dutra CL, Araújo CL, Bertoldi D. Prevalência de sobrepeso em adolescentes: um estudo de base populacional em uma cidade no Sul do Brasil. Cad Saúde Pública 2006; 22:151-62.

9. Proctor M, Moore L, Gao D, Cupples L, Bradlee M, Hood M, et al. Television viewing and change in body fat from preschool to early adolescence: the Framingham Children's Study. Int J Obes Relat Metab Disord 2003; 27:827-33.

10. Hancox R, Milne B, Poulton R. Association between child and adolescent television viewing and adult health: a longitudinal birth cohort study. Lancet 2004; 364:257-62.

11. Vereecken CA, Todd J, Roberts C, Mulvihill C, Maes L. Television viewing behaviour and associations with food habits in different countries. Public Health Nutr 2006; 9:244-50.

12. DietzW. Factors associated with childhood obesity. Nutrition 2002; 7:290-1.

13. Borzekowski DL, Robinson TN. The 30-second effect: an experiment revealing the impact of television commercials on food preferences of preschoolers. J Am Diet Assoc 2001; 101:42-6.

14. Utter J, Scragg R, Schaaf D. Associations between television viewing and consumption of commonly advertised foods among New Zealand children and young adolescents. Public Health Nutr 2006; 9:606-12.

15. Utter J, Neumark-Sztainer D, Jeffery R, Story M. Couch potatoes or french fries: are sedentary behaviors associated with body mass index, physical activity, and dietary behaviors among adolescents? J Am Diet Assoc 2003; 103:1298-305.
16. Coon KA, Goldberg J, Rogers BL, Tucker KL. Relationship between use of television during meals and children's food consumption patterns. Pediatrics 2001; 107:e7.

17. Campbell KJ, Crawford DA, Ball K. Family food environment and dietary behaviors likely to promote fatness in 5-6 year-old children. Int J Obes (Lond) 2006; 30:1272-80.

18. Ng C, Young TK, Corey PN. Associations of television viewing, physical activity and dietary behaviours with obesity in aboriginal and non-aboriginal Canadian youth. Public Health Nutr 2010; $13: 1430-7$

19. Rey-López JP, Vicente-Rodríguez G, Répásy J, Mesana MI, Ruiz JR, Ortega FB, et al. Food and drink intake during television viewing in adolescents: the Healthy Lifestyle in Europe by Nutrition in Adolescence (HELENA) study. Public Health Nutr 2011; 14:1563-9.

20. World Health Organization. Inequalities young people's health: key findings from the Health Behaviour in School-aged Children (HBSC) 2005/2006 survey fact sheet. Copenhagen: World Health Organization; 2008.

21. Toyran M, Ozmert E, Yurdakok K, Turk J. Television viewing and its effect on physical health of school age children. Turk J Pediatr 2002; 44:194-203.

22. Certain LK, Kahn RS. Prevalence, correlates, and trajectory of television viewing among infants and toddlers. Pediatrics 2002; 109:634-42.

23. Lindquist CH, Reynolds KD, Goran MI. Sociocultural determinants of physical activity among children. Prev Med 1999; 29:305-12.

24. van den Bulck J, van den Bergh B. The influence of perceived parental guidance patterns in children's media use: gender differences and media displacement. J Broadcast Elec Media 2000; 44:329-48.

25. Instituto Brasileiro de Geografia e Estatística. Pesquisa Nacional de Saúde Escolar. Rio de Janeiro: Instituto Brasileiro de Geografia e Estatística; 2009.

26. Malta DC, Sardinha LMV, Mendes I, Barreto SM, Giatti L, Castro IRR, et al. Prevalência de fatores de risco e proteção de doenças crônicas não transmissíveis em adolescentes: resultados da Pesquisa Nacional de Saúde do Escolar (PeNSE), Brasil, 2009. Ciênc Saúde Coletiva 2010; 15 Suppl 2: 3009-19.

27. Szwarcwald CL, Souza Júnior-PRB, Esteves MAP, Damacena GN, Viacava F. Socio-demographic determinants of self-rated health in Brazil. Cad Saúde Pública 2005; 21 Suppl 1:S54-64.

28. Grund A, Krause H, Siewers M, Rieckert H, Muller MJ. Is TV viewing an index of physical activity and fitness in overweight and normal weight children? Public Health Nutr 2001; 4:1245-51.

29. Jackson DM, Djafarian K, Stewart J, Speakman JR. Increased television viewing is associated with elevated body fatness but not with lower total en ergy expenditure in children. Am J Clin Nutr 2009; 89:1031-6. 
30. Manios Y, Kourlaba G, Kondaki K, Grammatikaki E, Anastasiadou A, Roma-Giannikou E. Obesity and television watching in preschoolers in Greece: the GENESIS study. Obesity (Silver Spring) 2009; 17:2047-53.

31. Drewnowski A, Darmon N. The economics of obesity: dietary energy density and energy cost. Am J Clin Nutr 2005; 82(1 Suppl):265S-73S.

32. Cairns G, Angus K, Hastings G. The extent, nature and effects of food promotion to children: a review of the evidence to December 2008. Geneva: World Health Organization; 2009.

33. Livingstone S, Helsper E. Advertising foods to children: understanding promotion in the context of children's daily lives. London: Department of $\mathrm{Me}-$ dia and Communications, London School of Economics and Political Science; 2004.

34. Almeida SS, Nascimento PCBD, Quaioti TCB. Quantidade e qualidade de produtos alimentícios anunciados na televisão brasileira. Rev Saúde Pública 2002; 36:353-5.

35. Monteiro CA, Levy RB, Claro RM, Castro IRR, Cannon G. Increasing consumption of ultra-processed foods and likely impact on human health: evidence from Brazil. Public Health Nutr 2011; 14:5-13.
36. Centers for Disease Control and Prevention. Health risk behaviors among adolescents who do and do not attend school: United States, 1992. MMWR Morb Mortal Wkly Rep 1994; 43:129-32.

37. Tresidder J, Macaskill P, Bennett D, Nutbeam D. Health risk and behaviour of out-of-school 16year-olds in New South Wales. Aust N Z J Public Health 1997; 21:168-74.

38. Kebede D, Alem A, Mitike1 G, Enquselassie F, Berhane F, Abebe Y, et al. Khat and alcohol use and risky sex behaviour among in-school and out-ofschool youth in Ethiopia. BMC Public Health 2005; 5:109.

39. Sychareun V, Thomsen S, Faxelid E. Concurrent multiple health risk behaviors among adolescents in Luangnamtha province, Lao PDR. BMC Public Health 2011; 11:36

40. Instituto Brasileiro de Geografia e Estatística. Pesquisa Nacional por Amostra de Domicílios 2008. Rio de Janeiro: Instituto Brasileiro de Geografia e Estatística; 2008.

Recebido em 17/Mar/2012

Aprovado em 07/Ago/2012 